\title{
Peroral Endoscopic Myotomy Is Expanding Its Indications
}

\author{
(J Am Coll Surg 2013;217:598-605)
}

\author{
Hee Man Kim \\ Department of Internal Medicine, Yonsei University Wonju College of Medicine, Wonju, Gangwon-do, Korea
}

\section{Summary}

Peroral endoscopic myotomy (POEM) is a novel non-surgical endoscopic procedure for treatment of achalasia, and has been performed as a primary therapy for achalasia with good short-term outcomes. Heller myotomy is a surgical therapy for treatment of achalasia and has a good long-term prognosis. However, symptoms of achalasia after Heller myotomy persist or recur with the rate of $3.5 \%$ to $15 \%{ }^{1}$

Recently, Onimaru et $\mathrm{al}^{2}$ have tested the feasibility of POEM as a rescue therapy in achalasia patients with persistent or recurrent symptoms after undergoing surgical myotomy. Of 315 patients receiving POEM in Showa University Northern Yokohama Hospital, 11 (3.5\%) patients had been treated with surgical myotomy as a primary therapy. They had complained persistent or recurrent symptoms of achalasia. Of these 11 patients, 10 patients underwent POEM as a rescue therapy. In 10 patients at 3 months after POEM, lower esophageal sphincter resting pressure $(22.1 \pm 6.6 \mathrm{mmHg}$ vs. $10.9 \pm 4.5 \mathrm{mmHg})$ was significantly reduced, and Eckardt symptom scores $(6.5 \pm 1.3$ vs. $1.1 \pm 1.3$ ) were also significantly improved. Adverse events related to POEM procedure in these patients were not reported.

\section{Comments}

The conventional modalities for treatment of achalasia include pharmacologic agents, pneumatic balloon dilatation, botulinum toxin injection and surgical myotomy. POEM is a newly-developed minimally-invasive modality, and it intends a permanent cure for achalasia. ${ }^{3}$ It is opening up a new chapter in treatment of achalasia, although the history of POEM is very short. The first preclinical experiments of endoscopic esophageal myotomy were performed in an animal model by Pasricha et $\mathrm{al}^{4}$ in the early 2000 s. Doctor Inoue firstly has applied POEM to human patients with achalasia, and his pioneering work has been proved effective until now, although follow-up periods are short. ${ }^{3,5}$ Around the same time, Doctor Zhou also has independently reported his own experience of POEM in patients. ${ }^{6}$ His results are consistent with those of Inoue et al Since then, POEM has been performed in several institutes of the world, and the short-term outcomes of POEM are very promising. The first prospective multicenter study performed at 5 centers in Europe and North America has shown that the treatment success rates at 6 months and 12 months were $88.5 \%$ and $82.4 \%$, respectively, during the mean follow-up period of 10.1 months. ${ }^{7}$ However long-term outcomes remain uncertain, and fur-

Received: November 28, 2013 Revised: None Accepted: November 30, 2013

(c) This is an Open Access article distributed under the terms of the Creative Commons Attribution Non-Commercial License (http://creativecommons. org/licenses/by-nc/3.0) which permits unrestricted non-commercial use, distribution, and reproduction in any medium, provided the original work is properly cited.

*Correspondence: Hee Man Kim, MD, PhD

Department of Internal Medicine, Yonsei University Wonju College of Medicine, 20 Ilsan-ro, Wonju, Gangwon-do 220-701, Korea

Financial support: None.

Tel: +82-33-741-1229, Fax: +82-33-741-1228, E-mail: loverkorea2009@gmail.com

Conflicts of interest: None. 
ther investigation is needed.

Recently, two comparative studies between POEM and Heller myotomy have been published. The one study has shown that treatment results of POEM were compatible to those of Heller myotomy, but POEM had the benefit of a shorter hospitalization than Heller myotomy (mean, 1.1 days vs. 2.2 days). ${ }^{8}$ In this study, 64 patients received Heller myotomy, and 37 patients received POEM, and both groups had similar improvements in Eckardt scores (mean, 1.7 vs. 1.2, respectively) at 6 months after treatment. Another small study composed of 17 patients undergoing Heller myotomy and 12 patients undergoing POEM has reported similar findings. ${ }^{9}$ Heller myotomy may be destined to be substituted with POEM as a first-line modality for treatment of achalasia.

The appropriate therapy for recurrence or persistent achalasia after Heller myotomy remains undetermined. Additional treatments such as pneumatic dilatation, redo Heller myotomy and esophagectomy have been tried, but the outcomes are controversial. One review article has reported a good response of redo Heller myotomy for failed Heller myotomy (approximately $86 \%$ ), compared with pneumatic dilatation, and another study has reported a substantial efficacy (approximately 80\%) of redo Heller myotomy. ${ }^{10,11}$

The current study by Inoue et $\mathrm{al}^{2}$ has suggested that POEM is an effective rescue therapy in patients who have persistent or recurrent symptoms of achalasia after Heller myotomy. During a mean period of 18.3 months (range, 3-31 months), all patients had improved clinical parameters. Coincidentally, these findings are compatible with those of another study by Zhou et $\mathrm{al}^{12}$ having reported that 11 patients of 12 patients with failed relief of symptom after Heller myotomy had successful outcome from POEM during a mean follow-up period of 10.4 months (range, 5-15 months). These 2 independent studies have shown the similar results favoring POEM as a secondary therapy after Heller myotomy. In addition, POEM is minimally-invasive, although the technique is somewhat difficult, and reduces hospitalization days. However, these two studies have common limitations: the small sample size and single-armed design. Considering the low prevalence of achalasia, the small sample size is not an obstacle to introducing the innovative treatment, and this problem will be resolved over time.

In addition, POEM is making progress, and even applied to the stomach. Recently, so-called gastric peroral myotomy is attempted to treat refractory gastroparesis in a human patient. ${ }^{13}$
The gastric peroral myotomy is an endoscopic pyloromytomy to improve gastroparesis symptoms.

In summary, POEM is an innovative therapeutic modality of achalasia, and it may become a substitute of Heller myotomy as a primary therapy and a rescue therapy of failed Heller myotomy in achalasia.

\section{References}

1. Petersen RP, Pellegrini CA. Revisional surgery after Heller myotomy for esophageal achalasia. Surg Laparosc Endosc Percutan Tech 2010;20:321-325.

2. Onimaru $\mathrm{M}$, Inoue $\mathrm{H}$, Ikeda $\mathrm{H}$, et al. Peroral endoscopic myotomy is a viable option for failed surgical esophagocardiomyotomy instead of redo surgical Heller myotomy: a single center prospective study. J Am Coll Surg 2013;217:598-605.

3. Inoue $\mathrm{H}$, Tianle $\mathrm{KM}$, Ikeda $\mathrm{H}$, et al. Peroral endoscopic myotomy for esophageal achalasia: technique, indication, and outcomes. Thorac Surg Clin 2011;21:519-525.

4. Pasricha PJ, Hawari R, Ahmed I, et al. Submucosal endoscopic esophageal myotomy: a novel experimental approach for the treatment of achalasia. Endoscopy 2007;39:761-764.

5. Inoue $\mathrm{H}$, Minami $\mathrm{H}$, Satodate $\mathrm{H}$, et al. First clinical experience of submucosal endoscopic myotomy for esophageal achalasia with no skin incision. Gastrointest Endosc 2009;69:AB122.

6. Zhou PH, Cai MY, Yao LQ, et al. [Peroral endoscopic myotomy for esophageal achalasia: report of 42 cases.] Zhonghua Wei Chang Wai Ke Za Zhi 2011;14:705-708. [Chinese]

7. Von Renteln D, Fuchs KH, Fockens P, et al. Peroral endoscopic myotomy for the treatment of achalasia: an international prospective multicenter study. Gastroenterology 2013;145:309-311.

8. Bhayani NH, Kurian AA, Dunst CM, Sharata AM, Rieder E, Swanstrom LL. A comparative study on comprehensive, objective outcomes of laparoscopic Heller myotomy with per-oral endoscopic myotomy (POEM) for achalasia. Ann Surg Published Online First: 28 Oct 2013. Doi: 10.1097/SLA.0000000000000268.

9. Teitelbaum EN, Rajeswaran S, Zhang R, et al. Peroral esophageal myotomy (POEM) and laparoscopic Heller myotomy produce a similar short-term anatomic and functional effect. Surgery 2013;154: 885-891.

10. Wang L, Li YM. Recurrent achalasia treated with Heller myotomy: a review of the literature. World J Gastroenterol 2008;14:7122-126.

11. Loviscek MF, Wright AS, Hinojosa MW, et al. Recurrent dysphagia after Heller myotomy: is esophagectomy always the answer? J Am Coll Surg 2013;216:736-743; discussion 743-744.

12. Zhou PH, Li QL, Yao LQ, et al. Peroral endoscopic remyotomy for failed Heller myotomy: a prospective single-center study. Endoscopy 2013;45:161-166.

13. Khashab MA, Stein E, Clarke JO, et al. Gastric peroral endoscopic myotomy for refractory gastroparesis: first human endoscopic pyloromyotomy (with video). Gastrointest Endosc 2013;78:764-768. 